\title{
Mechanism underlying the significant role of the miR-4262/SIRT1 axis in children with inflammatory bowel disease
}

\author{
XIAOZHI DENG, LIHONG SHANG, MIN DU, LAN YUAN, LIJING XIONG and XIAOLI XIE \\ Department of Children's Gastroenterology, Chengdu Women's and Children's Central Hospital, School of Medicine, \\ University of Electronic Science and Technology of China, Chengdu, Sichuan 611731, P.R. China
}

Received December 27, 2019; Accepted May 15, 2020

DOI: $10.3892 /$ etm. 2020.8918

\begin{abstract}
Inflammatory bowel disease (IBD) is a term used to describe chronic and recurrent gastrointestinal disease. In total, $>2$ million individuals worldwide have been diagnosed with IBD, including ulcerative colitis (UC), Crohn's disease (CD) and indeterminate colitis. There is accumulating evidence to indicate that microRNAs (miRNAs or miRs) are involved in the development and progression of IBD. miR-4262, an underlying promoter in tumor diseases, has been reported to regulate inflammatory responses. However, the potential mechanisms underlying the role of miR-4262 in IBD remain unknown. The present study attempted to explore the role and mechanisms of miR-4262 in IBD. Firstly, reverse transcription-quantitative PCR (RT-qPCR) was used to detect the expression of miR-4262 in 30 IBD colonic mucosa tissues, 30 normal tissues, $2 \%$ dextran sulfate sodium (DSS)-treated Caco- 2 cells and normal cells. It was demonstrated that the expression levels of miR-4262 in IBD colonic mucosa tissues and 2\% DSS-stimulated Caco- 2 cells were markedly higher compared with those in the control groups. Target gene prediction databases and dual-luciferase reporter assays were then used, and sirtuin 1 (SIRT1) was identified as a target gene of miR-4262. Furthermore, the levels of SIRT1 in 2\% DSS-stimulated Caco-2 cells and IBD colonic mucosa tissues were suppressed compared with the corresponding control groups. In addition, it was observed that miR-4262 negatively regulated SIRT1 expression in Caco-2 cells. Thereafter, Caco-2 cells were treated with inhibitor control, miR-4262 inhibitor, control-siRNA or SIRT1-siRNA for $48 \mathrm{~h}$, followed by $2 \%$ DSS treatment for 4 days. The secretion of inflammatory factors was analyzed via ELISA and RT-qPCR. MTT assay, flow
\end{abstract}

Correspondence to: Dr Xiaoli Xie, Department of Children's Gastroenterology, Chengdu Women's and Children's Central Hospital, School of Medicine, University of Electronic Science and Technology of China, 1617 Section One of Riyue Avenue, Qingyang, Chengdu, Sichuan 611731, P.R. China

E-mail: xiex112026@163.com

Key words: inflammatory bowel disease, microRNA, miR-4262, SIRT1 cytometry and western blot analysis were performed to assess cell viability, apoptosis and NF- $\kappa \mathrm{B}$ signaling pathway-related protein levels, respectively. The results indicated that DSS enhanced the inflammatory response, suppressed cell viability and promoted cell apoptosis, and this was decreased following transfection with an miR-4262 inhibitor. In addition, 2\% DSS upregulated p-p65 expression and enhanced the ratio of p-p65/p65, while the miR-4246 inhibitor exerted an opposite effect. All the effects of miR-4262 inhibitor on Caco- 2 cells were eliminated following transfection with SIRT1-siRNA. It was thus concluded that miR-4262 may serve a role in the progression of IBD via targeting SIRT1, and miR-4262/SIRT1 may represent a potential target for the diagnosis and treatment of IBD.

\section{Introduction}

Inflammatory bowel disease (IBD), a gastrointestinal tract disease, is regulated by the inflammatory response and is associated with symptoms such as gastrointestinal bleeding, colonic shortening, abdominal pain, diarrhea and colonic chronic inflammation $(1,2)$. Ulcerative colitis (UC) and Crohn's disease (CD) are the two main types of IBD (3). Recently, the inflammatory response has been demonstrated to accelerate the pathogenesis of IBD (4). Although numerous efforts have been made to elucidate the mechanisms underlying IBD, the exact pathogenesis and mechanisms of IBD remain unclear. Therefore, anti-inflammatory drugs and the recognition of specific IBD agents may serve as novel therapeutic approaches for IBD.

MicroRNAs (miRNAs or miRs) are small non-coding RNA molecules that can regulate target gene levels or cell behaviors by degrading mRNAs or restricting translational levels (5). Multiple reports have confirmed that the abnormal expression of miRNAs is highly associated with various diseases, including IBD (6-8). Moreover, certain miRNAs exhibit anti-inflammatory effects and can be used as potential therapeutic targets. For instance, Huang et al (9) reported that the inhibition of miRNA-210 suppresses the pro-inflammatory response and reduces acute brain injury due to ischemic stroke in mice. In addition, Kumar et al (10) demonstrated that miRNA-26a modulates the inflammatory response induced by Toll-like receptor 4 stimulation in microglia. miR-4262, a recently discovered miRNA, has been identified as a biomarker and has been demonstrated to serve a promotive 
role in various diseases, including breast cancer (11), colon cancer (12) and cutaneous malignant melanoma (13). However, whether miR-4262 influences the development of IBD has not yet been reported, to the best of our knowledge.

Sirtuin 1 (SIRT1) serves a role in a number of biological functions, such as the inflammatory responses, cell apoptosis and signaling pathway regulations $(14,15)$. However, whether SIRT1 serves a role in the progression of IBD and the underlying molecular mechanisms requires further elucidation. It has been indicated that dextran sodium sulfate (DSS) can be used to establish an intestinal in vitro barrier model due to its rapidity, simplicity and controllability $(16,17)$.

The present study attempted to explore the role and mechanisms of miR-4262 in IBD. The expression of miR-4262 in IBD colonic mucosa tissues, normal tissues, DSS-treated Caco-2 cells and normal Caco-2 cells was determined. The role of miR-4262 in DSS-induced inflammation was investigated in the intestinal epithelial cell line, Caco-2, and the possible mechanisms of action of miR-4262 in IBD were analyzed.

\section{Materials and methods}

Clinical tissue samples. Between December 2016 and December 2018, colonic mucosa tissues were collected from 30 children with IBD (15 males; 15 females; age range: 7-11 years; mean age: 9.4 years) and 30 children without IBD (15 males; 15 females; age range: 6-12 years; mean age: 8.8 years) at Chengdu Women's and Children's Central Hospital (Chengdu, China). Patients with infectious colitis and colorectal cancer were excluded. Liquid nitrogen was employed to preserve the specimens at $-80^{\circ} \mathrm{C}$ until use. All specimens were obtained with written informed consent and the present study was approved by the Ethics Committee of Chengdu Women's and Children's Central Hospital.

Caco-2 cell culture and DSS treatment. The normal intestinal epithelial cell line Caco-2 was purchased from American Type Culture Collection. The cells were cultured in Dulbecco's modified Eagle medium (DMEM)/F12 (Gibco; Thermo Fisher Scientific, Inc.) containing $10 \%$ fetal calf serum (FBS; HyClone; GE Healthcare Life Sciences) and 1\% penicillin-streptomycin and were maintained at $37^{\circ} \mathrm{C}$ with $5 \%$ $\mathrm{CO}_{2}$ in an incubator. Caco-2 cells were treated with $2 \%$ DSS at $37^{\circ} \mathrm{C}$ for 4 days to construct the inflammatory model (18).

Reverse transcription-quantitative PCR (RT-qPCR). SIRT1, inflammatory cytokines [interleukin (IL)-1 $\beta$, IL-6 and tumor necrosis factor (TNF)- $\alpha$ ] and miR-4262 levels were measured via RT-qPCR. The isolation of RNA from colonic mucosa tissues and Caco-2 cells was performed using the RNA-isolation kit (Thermo Fisher Scientific, Inc.) according to the manufacturer's instructions. cDNA was obtained using a PrimeScript RT kit (Takara Bio, Inc.) according to the manufacturer's protocol, followed by PCR amplification on an ABI Prism 7500 system (Applied Biosystems; Thermo Fisher Scientific, Inc.) with SYBR Premix Ex-Taq (Takara Bio, Inc.). miRNA and mRNA expression were normalized to U6 and GAPDH, respectively. The reaction conditions were as follows: Initial denaturation at $95^{\circ} \mathrm{C}$ for $5 \mathrm{~min} ; 30$ cycles of $94^{\circ} \mathrm{C}$ for $30 \mathrm{sec}, 60^{\circ} \mathrm{C}$ for $30 \mathrm{sec}$ and $72^{\circ} \mathrm{C}$ for $1 \mathrm{~min}$; and a final extension at $72^{\circ} \mathrm{C}$ for $5 \mathrm{~min}$. Primers were produced by Sangon Biotech Co., Ltd. and the sequences were as follows: miR-4262 forward, 5'-TGCGGGACATTCAGA-3' and reverse, 5'-CCAGTGCAGGGTCCGAGGT-3'; SIRT1 forward, 5'-AAT CCAGTCATTAAAGGTCTACAA-3' and reverse, 5'-TAG GACCATTACTGCCAGAGG-3'; TNF- $\alpha$ forward, 5'-GAA CTGGCAGAAGAGGCACT-3' and reverse 5'-GGTCTG GGCCATAGAACTGA-3'; IL-1 $\beta$ forward, 5'-TGTGAAATG CCACCTTTTGA-3' and reverse, 5'-TGAGTGATACTGCCT GCCTG-3'; IL-6 forward, 5'-CCGGAGAGGAGACTTCAC AG-3' and reverse, 5'-CAGAATTGCCATTGCACA-3'; U6 forward, 5'-GCTTCGGCAGCACATATACTAAAAT-3' and reverse, 5'-CGCTTCACGAATTTGCGTGTCAT-3'; and GAPDH forward, 5'-CTTTGGTATCGTGGAAGGACTC-3' and reverse, 5'-GTAGAGGCAGGGATGATGTTCT-3'. The relative results were analyzed using the $2^{-\Delta \Delta \mathrm{Cq}}$ method (19).

Cell transfection. The miR-4262 inhibitor and inhibitor control were synthesized by Shanghai Genepharma Co. Ltd. Caco-2 cells were cultured to 70-80\% confluency and transiently transfected with $100 \mathrm{nM}$ miR-4262 inhibitor (cat no. HSTUD1216; Sigma-Aldrich; Merck KGaA), $100 \mathrm{nM}$ inhibitor control (cat no. HMC0002; Sigma-Aldrich; Merck KGaA), $0.2 \mu \mathrm{M}$ control-siRNA (cat no. sc-36869; Santa Cruz Biotechnology, Inc.) or $0.2 \mu \mathrm{M}$ SIRT1-siRNA (cat no. sc-40986; Santa Cruz Biotechnology, Inc.) using Lipofectamine 2000 (Thermo Fisher Scientific, Inc.) for $48 \mathrm{~h}$, according to the manufacturer's instructions. Following transfection, the cells were stimulated with $2 \% \mathrm{DSS}$ at $37^{\circ} \mathrm{C}$ for 4 days for further analysis.

Dual-luciferase reporter assay. TargetScan 7.2 (http://www. targetscan.org/vert_72/) was adopted to investigate the putative binding sites. The data suggested that SIRT1 was a potential target of miR-4262. Subsequently, the miR-4262 binding sites on SIRT1 3'-UTR, including wild-type or mutant, were inserted into the pGL-control vector (Promega Corporation) to construct a wild-type SIRT1 plasmid (SIRT1-WT) or SIRT1 mutation plasmid (SIRT1-MUT), respectively. The mimic control or miR-4262 mimic (Shanghai GenePharma Co., Ltd.) were then co-transfected with luciferase reporter vectors into Caco-2 cells using Lipofectamine 2000 (Invitrogen; Thermo Fisher Scientific, Inc.) for $48 \mathrm{~h}$, according to the manufacturer's protocol. The luciferase activity was assessed using the Dual-Luciferase Reporter assay system (Promega Corporation) following the manufacturer's protocol. All luciferase activities were normalized to Renilla luciferase activity.

Enzyme-linked immunosorbent assay (ELISA). ELISAs (all Beyotime Institute of Biotechnology) was adopted to measure the levels of TNF- $\alpha$ (cat. no. PT518), IL-6 (cat. no. PI330) and IL-1 $\beta$ (cat. no. PI305) in the cell supernatant following treatment. The optical density (OD) value of each well at $450 \mathrm{~nm}$ was detected, according to the manufacturer's instructions.

MTT assay. Cell viability was evaluated via MTT assay. The Caco- 2 cells were cultured in 96 -well plates at $37^{\circ} \mathrm{C}$, and the cells were then transfected with miR-4262 inhibitor, inhibitor control, control-siRNA and SIRT1-siRNA for $48 \mathrm{~h}$ and treated with $2 \% \operatorname{DSS}(18)$ at $37^{\circ} \mathrm{C}$ for a further 4 days. Following treatment, $10 \mu \mathrm{l}$ MTT solution (Amresco LLC) was 
added to the cells, followed by incubation at $37^{\circ} \mathrm{C}$ for $4 \mathrm{~h}$, and DMSO (Sangon Biotech Co., Ltd.) was then used to dissolve the formazan crystals. Finally, the OD values at $490 \mathrm{~nm}$ were detected using a multifunctional plate reader (BD Biosciences).

Flow cytometric analysis. Caco-2 cell apoptosis was detected via flow cytometric analysis. In the present study, the cells were transfected with miR-4262 inhibitor, inhibitor control, control-siRNA and SIRT1-siRNA for $48 \mathrm{~h}$ and treated with $2 \%$ DSS for 4 days. Subsequently, the cells were trypsinized at room temperature for $1 \mathrm{~min}$ and stained with Annexin V-FITC/propidium iodide (PI; Beyotime Institute of Biotechnology), according to the manufacturer's protocol. The results were analyzed using a FACSCalibur flow cytometer (BD Biosciences) and FlowJo software (version 7.6.1; Tree Star, Inc.).

Western blot analysis. Total proteins from Caco-2 cells were extracted using RIPA buffer (Invitrogen; Thermo Fisher Scientific, Inc.) and quantified using a BCA Protein assay kit (Invitrogen; Thermo Fisher Scientific, Inc.). The denatured proteins were loaded on $10 \%$ SDS-PAGE, and transferred to PVDF membranes (Bio-Rad Laboratories, Inc.). After blocking with 5\% skim milk in PBST at room temperature for $90 \mathrm{~min}$, the membranes were cultured with primary antibodies against GAPDH (cat no. G9545), SIRT1 (cat no. S5447), phosphorylated (p)-p65 (cat no. SAB4504488) and p65 (cat no. SAB4502609; all from Sigma-Aldrich; Merck KGaA; 1:1,000) overnight at $4^{\circ} \mathrm{C}$. Horesradish peroxidase-conjugated oat anti-rabbit immunoglobulin $\mathrm{G}$ secondary antibody (cat no. ab7090; Abcam; 1:2,000) was incubated with the membranes at room temperature for 90 min. Finally, the results were quantified using Enhanced Chemiluminescence detection system reagents (EMD Millipore), according to the manufacturer's instructions.

Statistical analysis. GraphPad Prism 6 software (GraphPad Software, Inc.) was used for statistical analyses. All results are expressed as the mean \pm standard deviation from three independent experiments. Group comparisons between multiple groups were performed by one-way ANOVA followed by Tukey's post hoc test. Unpaired Student's t-test was used to make comparisons between two groups. $\mathrm{P}<0.05$ was considered to indicate a statistically significant difference.

\section{Results}

miR-4262 is upregulated in 2\% DSS-stimulated Caco-2 cells and IBD colonic mucosa tissues. To analyze the role of miR-4262 in IBD, the expression of miR-4262 in 30 IBD colonic mucosa tissues and 30 normal colonic mucosa tissues was determined via RT-qPCR. As presented in Fig. 1A, miR-4262 expression was significantly increased in the colonic mucosa tissues from children with IBD compared with the normal tissues. Furthermore, a higher miR-4262 expression was found in the 2\% DSS-induced Caco- 2 cells compared with the control (Fig. 1B). These data demonstrated that miR-4262 was upregulated in colonic mucosa tissues from patients with IBD and in 2\% DSS-stimulated Caco-2 cells and may thus be a regulator of IBD.
Prediction of SIRT1 as a direct target of miR-4262. miRNAs perform biological functions by mediating targets levels (5). Based on TargetScan 7.2 analysis, the role of miR-4262 in IBD was identified. As presented in Fig. 2A, SIRT1 was identified as a potential target of miR-4262. To further verify the hypothesis, a dual luciferase reporter system was conducted to explore the relevance between miR-4262 and SIRT1. Primarily, it was confirmed that miR-4262 mimic significantly enhanced miR-4262 levels in Caco-2 cells (Fig. 2B). A significant suppression in SIRT1 3'-UTR wild-type luciferase activity was observed compared with the mimic control, while SIRT1-MUT luciferase activity exhibited no significant differences (Fig. 2C). In summary, these data confirmed that SIRT1 represents a direct target of miR-4262.

Expression of SIRT1 is suppressed in 2\% DSS-stimulated Caco-2 cells and colonic mucosa tissues from patients with IBD. According to the aforementioned resukts, it was speculated that SIRT1 may participate in the progression of IBD. To confirm this assumption, RT-qPCR analysis was performed to detect the SIRT1 levels in colonic mucosa tissues from patients with IBD and in normal colonic mucosa tissues. The results suggested that SIRT1 mRNA expression was markedly decreased in tissues from patients with IBD compared with normal tissues (Fig. 3A). Moreover, the results from RT-qPCR and western blot analysis revealed that SIRT1 was downregulated in the 2\% DSS-treated Caco- 2 cells compared with the control cells (Fig. 3B and C). Thus, from these data, it can be concluded that SIRT1 participates in the development of IBD.

SIRTI-siRNA reverses the effects of miR-4262 inhibitor on SIRT1 expression in Caco-2 cells. Subsequently, it was determined whether miR-4262 affects SIRT1 expression in IBD. Inhibitor control, miR-4262 inhibitor, control-siRNA or SIRT1-siRNA were transfected into Caco-2 cells for $48 \mathrm{~h}$. RT-qPCR analysis suggested that compared with the inhibitor control group, miR-4262 was downregulated in Caco-2 cells following transfection with miR-4262 inhibitor (Fig. 4A). Moreover, as presented in Fig. 4B, compared with the control-siRNA group, SIRT1-siRNA significantly decreased the SIRT1 levels. To further explore the association between miR-4262 and SIRT1, RT-qPCR and western blot analysis were performed to measure SIRT1 expression in the different groups following transfection. Higher mRNA and protein expression levels of SIRT1 were identified in the miR-4262 inhibitor group; however, these effects were partially restored in the miR-4262 inhibitor + SIRT1-siRNA group compared with the miR-4262 inhibitor + control-siRNA group (Fig. 4C and D). These results further confirmed that SIRT1 represents a target gene of miR-4262 and that miR-4262 negatively regulates SIRT1 in IBD cells.

miR-4262 inhibitor decreases the secretion of inflammatory factors in DSS-stimulated Caco-2 cells via regulating SIRT1. Multiple reports have confirmed that IBD is associated with inflammatory factor secretion $(20,21)$. Thus, the levels of inflammatory cytokines were measured in DSS-treated Caco-2 cells following transfection for $48 \mathrm{~h}$. The results were verified using ELISAs. It was revealed that DSS promoted the release 

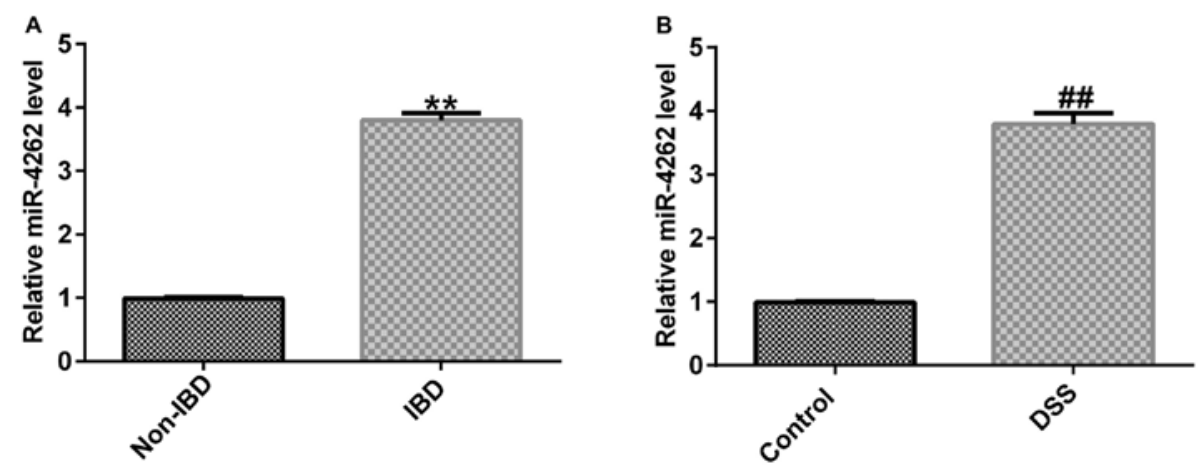

Figure 1. miR-4262 is significantly upregulated in colonic mucosa tissues from patients with IBD and DSS-treated Caco-2 cells. (A) The level of miR-4262 in IBD colonic mucosa tissues and normal colonic mucosa tissues was measured via RT-qPCR. (B) RT-qPCR analysis was performed to detect the miR-4262 mRNA levels in $2 \%$ DSS-treated Caco-2 cells and a normal intestinal epithelial cell line. The data are presented as the mean \pm SD. ${ }^{* *} \mathrm{P}<0.01$ vs. non-IBD; ${ }^{\#} \mathrm{P}<0.01$ vs. control. RT-qPCR, reverse transcription-quantitative PCR; miR, microRNA; IBD, inflammatory bowel disease; DSS, dextran sulfate sodium.
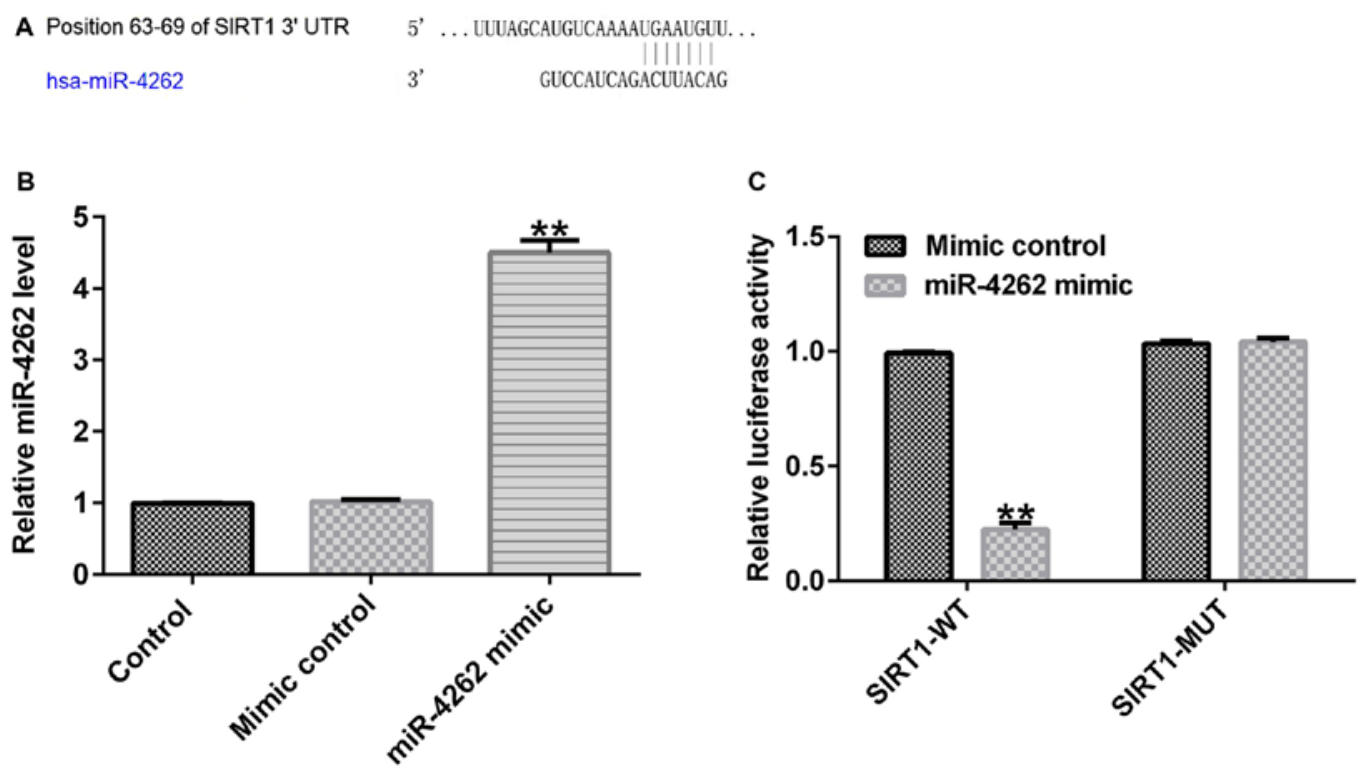

Figure 2. SIRT1 is a target gene of miR-4262. (A) A schematic of miR-4262 target site in SIRT1 3'untranslated region. (B) Expression level of miR-4262 in Caco-2 cells transfected with mimic control or miR-4262 mimic. (C) The dual luciferase reporter assay revealed the combination of miR-4262 mimics/control and WT/MUT SIRT1. The data are presented as the mean \pm SD. ${ }^{* *} \mathrm{P}<0.01$ vs. mimic control. WT, wild-type; MUT, mutant; miR, micro RNA; SIRT 1, sirtuin 1.

of TNF- $\alpha$, IL-1 $\beta$ and IL-6 in Caco-2 cells compared with the control group (Fig. 5A-C). In addition, cells cultured with DSS and miR-4262 inhibitor exhibited a lower inflammatory response, verified by the decreased TNF- $\alpha$, IL- $1 \beta$ and IL- 6 expression levels compared with the DSS + inhibitor control group. However, these effects were partially reversed following transfection with SIRT1-siRNA, and it was revealed that the levels of TNF- $\alpha$, IL-1 $\beta$ and IL- 6 were significantly enhanced in the DSS + miR-4246 inhibitor + SIRT1-siRNA group (Fig. 5A-C). The same trends were observed at the mRNA level via RT-qPCR (Fig. 5D-F). The aforementioned results demonstrated that SIRT1-siRNA reversed the inhibitory function of the miR-4262 inhibitor on the expression of inflammatory factors in DSS-treated Caco-2 cells, suggesting that the silencing of SIRT1 may prevent miR-4262 inhibitor from inhibiting the inflammatory response in DSS-induced Caco-2 cells.

miR-4262 inhibitor promotes cell viability and decreases cell apoptosis in DSS-stimulated Caco-2 by regulating SIRT1. To intensively explore the functional mechanism of miR-4262 in IBD, Caco-2 cells were transfected with inhibitor control, miR-4262 inhibitor, control-siRNA or SIRT1-siRNA for $48 \mathrm{~h}$, followed by $2 \%$ DSS treatment for 4 days. MTT assays and flow cytometric analysis were conducted to evaluate the Caco-2 cell viability and apoptosis, respectively. As presented in Fig. 6A, DSS treatment markedly inhibited Caco- 2 cell viability compared with the control. Moreover, in the DSS-induced cells, miR-4262 inhibitor stimulated cell viability compared to the inhibitor control. However, in the DSS + miR-4246 inhibitor + SIRT1-siRNA group, cell viability was significantly suppressed. As indicated in Fig. 6B and $\mathrm{C}$, it was demonstrated that compared with the control group, DSS significantly induced Caco-2 cell apoptosis, while compared with the DSS + inhibitor control group, transfection with a miR-4246 inhibitor markedly inhibited cell apoptosis; however, this effect was reversed in the DSS + miR-4246 inhibitor + SIRT1-siRNA group compared with the DSS + miR-4246 inhibitor + control-siRNA group. 
A

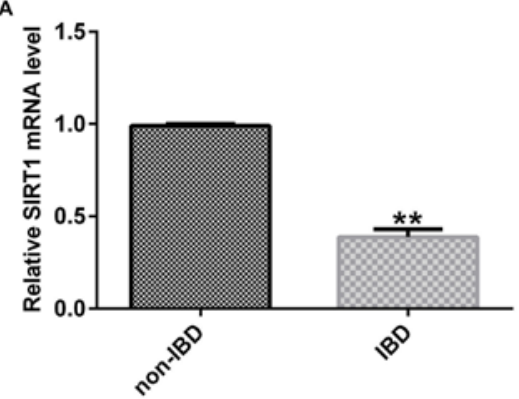

B

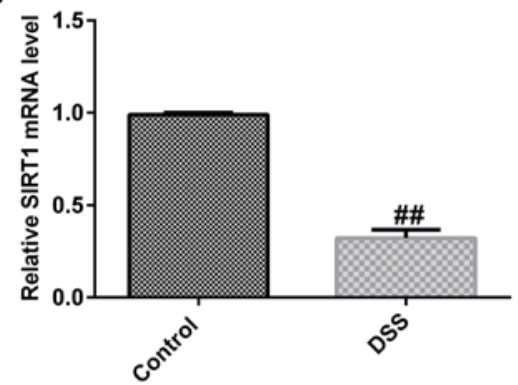

c

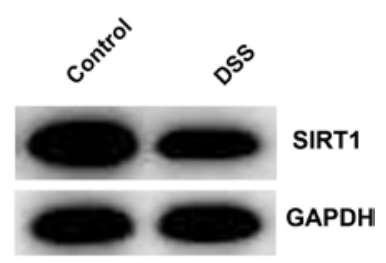

Figure 3. The expression of SIRT1 is suppressed in colonic mucosa tissues from patients with IBD and in DSS-treated Caco-2 cells. (A) RT-qPCR revealed the SIRT1 mRNA levels in IBD colonic mucosa tissues and normal colonic mucosa tissues. The mRNA levels and protein expression of SIRT1 were determined via (B) RT-qPCR and (C) western blot analysis in normal cells and 2\% DSS treated Caco-2 cells. Three independent experiment were conducted, and the data are presented as the mean $\pm \mathrm{SD}$. ${ }^{* *} \mathrm{P}<0.01$ vs. non-IBD; ${ }^{* \#} \mathrm{P}<0.01$ vs. control. RT-qPCR, reverse transcription-quantitative PCR; miR, microRNA; IBD, inflammatory bowel disease; DSS, dextran sulfate sodium; SIRT1, sirtuin 1.

A
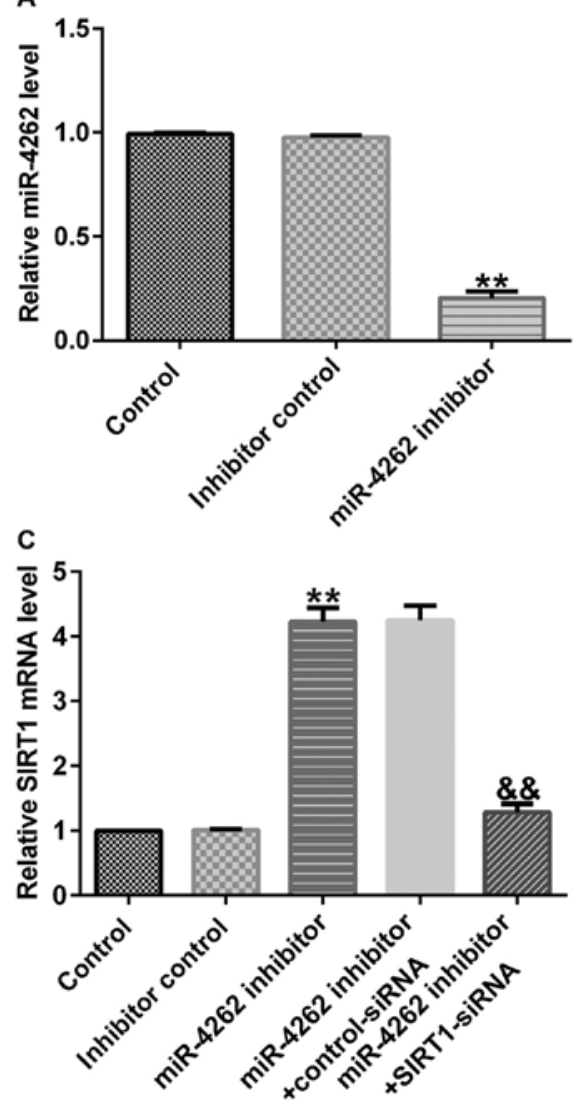

B

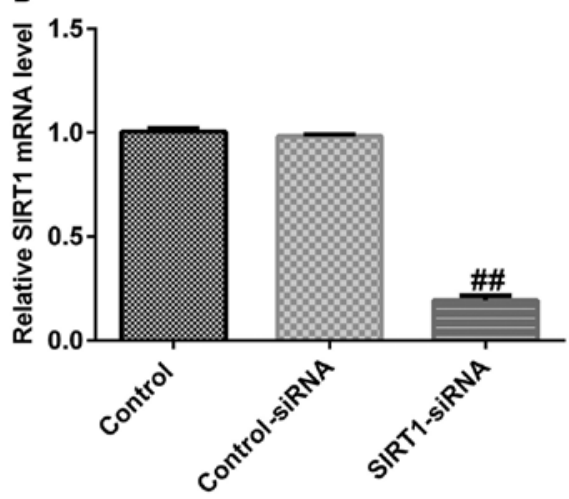

D

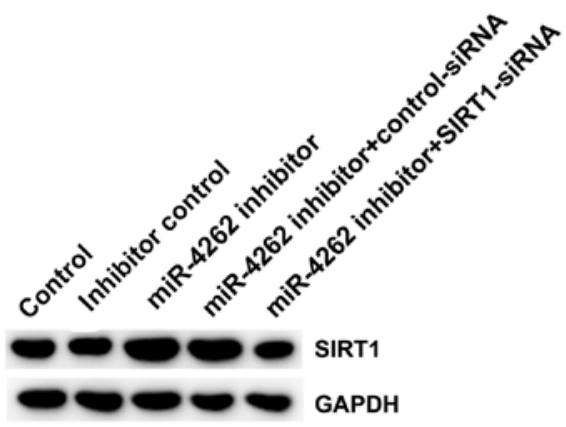

Figure 4. SIRT1-siRNA reverses the effects of miR-4262 inhibitor on SIRT1 expression. Control-siRNA, SIRT1-siRNA, inhibitor control or miR-4262 inhibitor were transfected into Caco-2 cells for $48 \mathrm{~h}$. Following transfection, RT-qPCR was performed to verify the transfection efficiency. (A) mRNA levels of miR-4262 following the transfection of miR-4262 inhibitor and inhibitor control in Caco-2 cells. (B) mRNA level of SIRT1 in Caco-2 cells following the transfection of control-siRNA and SIRT1-siRNA. The (C) mRNA and (D) protein level of SIRT1 were detected via RT-qPCR and western blot analysis, respectively, in Caco-2 cells following transfection with miR-4262 inhibitor + control-siRNA and miR-4262 inhibitor + SIRT1-siRNA. Three independent experiment were conducted, and the data are presented as the mean $\pm \mathrm{SD} .{ }^{* *} \mathrm{P}<0.01$ vs. inhibitor control; ${ }^{\# /} \mathrm{P}<0.01$ vs. control-siRNA; \&\& $\mathrm{P}<0.01$ vs. miR-4262 inhibitor + control-siRNA. RT-qPCR, reverse transcription-quantitative PCR; miR, microRNA; siRNA, small interfering RNA; DSS, dextran sulfate sodium; SIRT1, sirtuin 1.

miR-4262 inhibitor suppresses $N F-\kappa B$ pathway activation in DSS-stimulated Caco-2 cells cia regulating SIRT1. The $\mathrm{NF}-\kappa \mathrm{B}$ pathway has been confirmed to participate in the progression of IBD (22). Finally, western blot analysis was performed to detect the associated protein expression levels of the NF- $\kappa \mathrm{B}$ pathway in DSS-induced cells. The results revealed that p-p65 expression was increased (Fig. 7A) and the ratio of p-p65/p65 was enhanced (Fig. 7B) in the DSS-treated cells. However, it was then observed that p-p65 was downregulated and the ratio of p-p65/p65 was decreased in the DSS-treated Caco-2 cells following transfection with a miR-4262 inhibitor. However, in the DSS + miR-4246 inhibitor + SIRT1-siRNA 

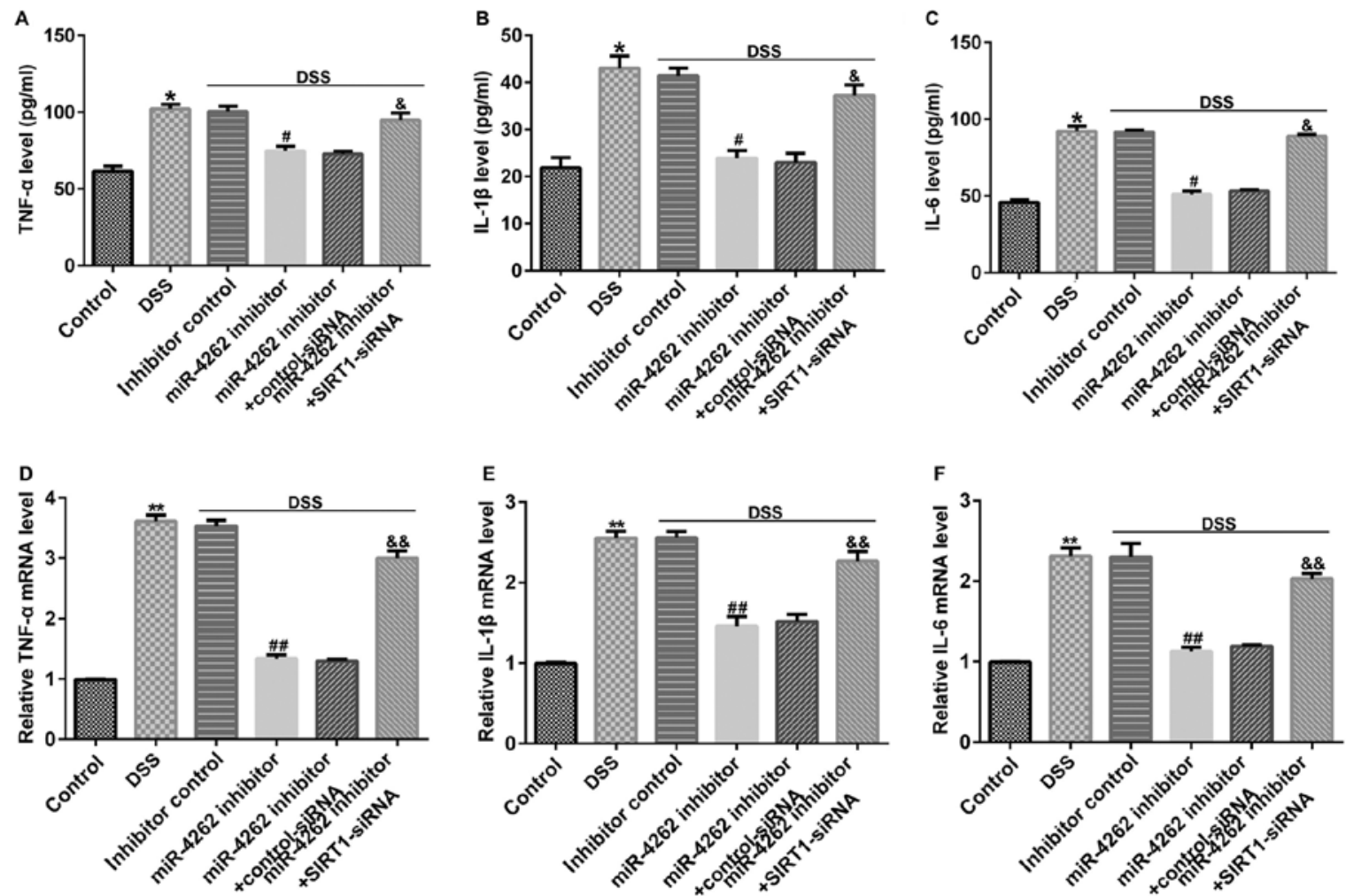

Figure 5. SIRT1-siRNA reverses the effects of miR-4262 inhibitor on the secretion of inflammatory factors. Inhibitor control, miR-4262 inhibitor, control-siRNA and SIRT1-siRNA were transfected into Caco- 2 cells for $48 \mathrm{~h}$. Then, the cells were stimulated with DSS for 4 days. Cells were divided into 6 groups: Control, DSS, DSS + inhibitor control, DSS + miR-4246 inhibitor, DSS + miR-4246 inhibitor + control-siRNA, DSS + miR-4246 inhibitor + SIRT1-siRNA (The release of the inflammatory factors (A) TNF- $\alpha$, (B) IL-1 $\beta$ and (C) IL- 6 were detected via ELISAs. The (D) TNF- $\alpha$, (E) IL-1 $\beta$ and (F) IL-6 mRNA levels were evaluated via reverse transcription-quantitative PCR in 6 groups. Three independent experiment were conducted, and the data are presented as the mean \pm SD. ${ }^{*} \mathrm{P}<0.05,{ }^{* *} \mathrm{P}<0.01$ vs. control; ${ }^{*} \mathrm{P}<0.05,{ }^{\# / t} \mathrm{P}<0.01$ vs. DSS + inhibitor control; ${ }^{\&} \mathrm{P}<0.05$, ${ }^{\text {\& }} \mathrm{P}<0.01$ vs. DSS + miR-4246 inhibitor + control-siRNA. miR, microRNA; siRNA, small interfering RNA; DSS, dextran sulfate sodium; SIRT1, sirtuin 1; IL, interleukin; TNF, tumor necrosis factor.

group, the expression of p-p65 was enhanced and the ratio of p-p65/p65 was increased. Collectively, the current results demonstrate that transfection with a miR-4262 inhibitor exerts protective effects on IBD cells by inhibiting both the inflammatory response and Caco-2 cell apoptosis via the regulation of SIRT1, indicating that miR-4262 may represent a potential target for IBD treatment.

\section{Discussion}

IBD, a chronic and immune disease, is associated with hindering intestinal peristalsis, a shortening colon and inflammation (23). A previous associated study indicated that chronic inflammation is a main risk factor in the progression of colorectal cancer and is involved in the pathogenesis of IBD, while therapies for patients with IBD are limited, where many patients do not respond to or cannot sustain treatment with these drugs, which have various side effects (24). Therefore, the present study aimed to identify a novel target and to explore the underlying mechanisms for IBD treatment. DSS is widely adopted to stimulate colonic inflammation for in vivo and in vitro IBD models $(25,26)$, and in a DSS mouse IBD model, DSS appears to be directly toxic to colonic epithelial cells in the basal crypt (26). Thus, in the present study, DSS was used to treat Caco-2 cells and the effects of DSS on Caco-2 cells and IBD tissues were examined.
It has been previously characterized that miRNAs serve various roles in disease development (27). Moreover, several miRNAs, such as miR-135a have been confirmed to participate in the progression of IBD (28). miR-4262, a recently discovered miRNA, functions as either an oncogene or tumor-suppressor gene in cancers (29). Numerous studies have demonstrated that miR-4262 exhibits an abnormal expression in multiple pathological processes, such as non-small cell lung cancer. For example, Sun et al (30) reported that miR-4262 was involved in paclitaxel resistance via the regulation of PTEN in non-small cell lung cancer. However, the findings by Song et al (31) indicated that the levels of miR-4262 were significantly decreased in osteosarcoma tissues. All these findings suggest that miR-4262 serves a vital role in the development of cancers. However, as far as is known, the specific mechanisms of action of miR-4262 in IBD remain to be clarified. In the current study, the effects of miR-4262 on the progression of IBD were investigated.

In the present study, the level of miR-4262 in IBD colonic mucosa tissues and normal tissues, as well as in $2 \%$ DSS-stimulated Caco-2 cells and untreated Caco-2 cells was first determined. It was revealed that the expression of miR-4262 was significantly higher in IBD colonic mucosa tissues compared with normal tissues. Moreover, a significant downregulation of miR-4262 in 2\% DSS-stimulated Caco-2 cells was observed, compared with normal cells. These results indicated that miR-4262 may represent a regulator of IBD. 
A

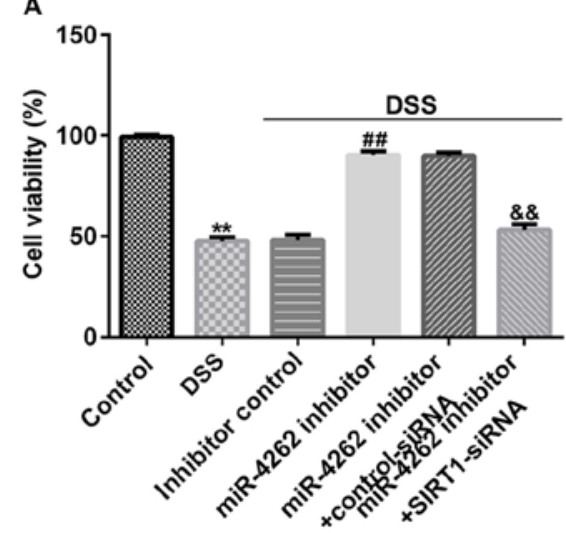

C

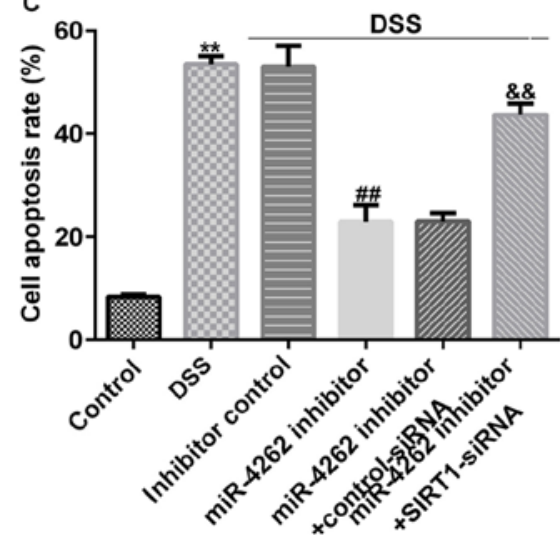

B
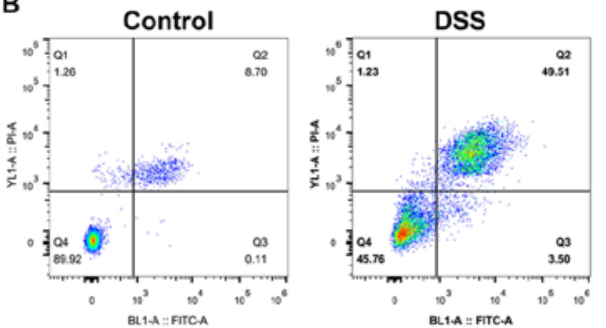

DSS+miR-4262 inhibitor DSS+miR-4262 inhibitor

DSS+miR-4262 inhibitor

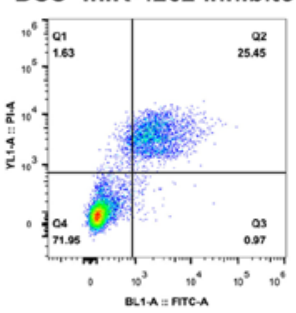

DSS+inhibitor control
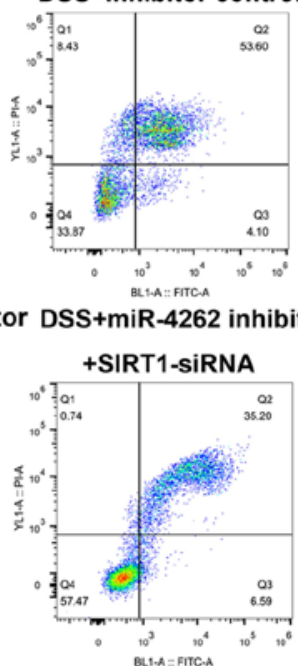

Figure 6. SIRT1-siRNA eliminates the effects of miR-4262 inhibitor on Caco-2 cell viability and apoptosis. Caco-2 cells were transfected with inhibitor control, miR-4262 inhibitor, control-siRNA and SIRT1-siRNA for $48 \mathrm{~h}$, and the cells were then treated with 2\% DSS for 4 days. Cells were divided into 6 groups: Control, DSS, DSS + inhibitor control, DSS + miR-4246 inhibitor, DSS + miR-4246 inhibitor + control-siRNA, DSS + miR-4246 inhibitor + SIRT1-siRNA (A) MTT was performed to detect Caco-2 cell viability in the 6 groups. (B) FCM was conducted to measure Caco-2 cell apoptosis in the 6 groups. (C) Results of FCM are displayed as a bar graph. Three independent experiment were conducted, and the data are presented as the mean $\pm \mathrm{SD}$. ${ }^{* *} \mathrm{P}<0.01 \mathrm{vs}$. Control; ${ }^{\# \#} \mathrm{P}<0.01$ vs. DSS + inhibitor control; \&\& $\mathrm{P}<0.01$ vs. DSS + miR-4246 inhibitor + control-siRNA. miR, microRNA; siRNA, small interfering RNA; DSS, dextran sulfate sodium; SIRT1, sirtuin 1; PI, propidium iodide.

A

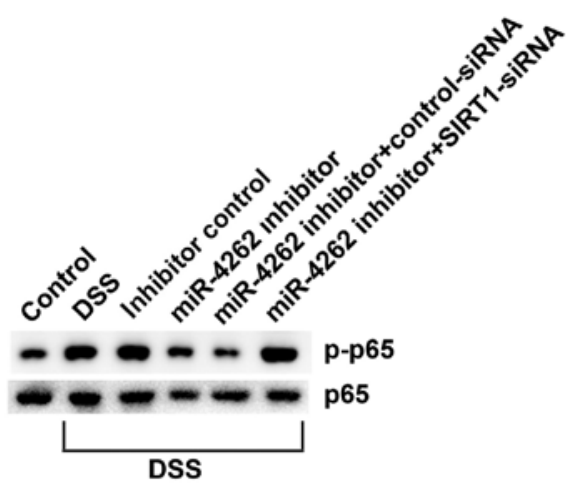

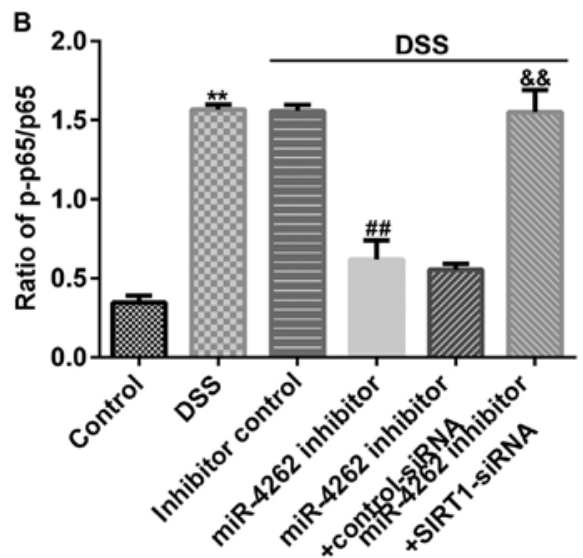

Figure 7. miR-4262 inhibitor alleviates the activation of the NF-KB pathway in Caco-2 cells and SIRT1-siRNA reverses the effects. Caco-2 cells were transfected with inhibitor control, miR-4262 inhibitor, control-siRNA and SIRT1-siRNA for $48 \mathrm{~h}$, and DSS was used to induce the cells for 4 days. Cells were divided into 6 groups: Control, DSS, DSS + inhibitor control, DSS + miR-4246 inhibitor, DSS + miR-4246 inhibitor + control-siRNA, DSS + miR-4246 inhibitor + SIRT1-siRNA. (A) Western blot analysis of p65 and p-p65 expression in different groups. (B) Relative protein expression of p-p65/p65 was measured. Three independent experiment were conducted, and the data are presented as the mean \pm SD. ${ }^{* *} \mathrm{P}<0.01 \mathrm{vs}$. control; ${ }^{* \#} \mathrm{P}<0.01 \mathrm{vs}$. DSS + inhibitor control; ${ }^{\&}{ }^{\mathrm{P}}<0.01$ vs. DSS + miR-4246 inhibitor + control-siRNA. miR, microRNA; siRNA, small interfering RNA; DSS, dextran sulfate sodium; SIRT1, sirtuin 1; $\mathrm{p}-$, phosphorylated.

Bioinformatics tools and a dual-luciferase reporter assay were then adopted to predict the miR-4262 target gene. The results indicated that miR-4262 directly targeted the SIRT1 3'-UTR and negatively regulated SIRT1 expression levels. It was 
demonstrated that the level of SIRT1 in IBD colonic mucosa tissues was higher compared with in normal colonic mucosa tissues. The results suggested that SIRT1 mRNA expression was significantly reduced in IBD tissues compared with normal tissues. In addition, the results from RT-qPCR and western blot analysis indicated that SIRT1 was downregulated in the 2\% DSS-treated Caco-2 cells compared with the control cells, suggesting that SIRT1 participated in the development of IBD. Based on these data, it was assumed that the altered expression of miR-4262 may affect the development of IBD. Furthermore, whether miR-4262 influences SIRT1 expression in IBD was determined. Inhibitor control, miR-4262 inhibitor, control-siRNA or SIRT1-siRNA were transfected into Caco- 2 cells for $48 \mathrm{~h}$. The data revealed that miR-4262 was downregulated in Caco-2 cells following transfection with miR-4262 inhibitor, and SIRT1-siRNA significantly decreased the SIRT1 levels. Moreover, higher mRNA and protein expression level of SIRT1 were obtained in the miR-4262 inhibitor group; however, these effects were abolished in the miR-4262 inhibitor + SIRT1-siRNA group. These results further confirmed that miR-4262 negatively regulates SIRT1 expression via targeting the 3 'UTR of SIRT1 in IBD.

Various reports have confirmed that the inflammatory response is a main characteristic of IBD and is highly associated with the progression of IBD $(20,21,32)$. Pro-inflammatory factors, including IL- $1 \beta$, TNF- $\alpha$ and IL- 6 have been confirmed as important regulators in the occurrence of IBD (25). DSS-induced murine and cell models of IBD are often adopted for the investigation of intestinal inflammatory diseases $(18,33)$. To further investigate the underlying mechanisms of miR-4262 in IBD, in the present study, Caco-2 cells were transfected with inhibitor control, miR-4262 inhibitor, control-siRNA or SIRT1-siRNA for $48 \mathrm{~h}$, followed by $2 \%$ DSS treatment for 4 days. It was demonstrated that DSS induced a greater production of pro-inflammatory factors in Caco-2 cells, while miR-4262 inhibitor alleviated the release of DSS-stimulated inflammatory factors and that these results were reversed by transfection with SIRT1-siRNA. DSS inhibited Caco-2 cell growth and induced cell apoptosis compared with the control. Meanwhile, miR-4262 inhibitor promoted cell growth and decreased cell apoptosis in DSS-induced Caco-2 cells, and this effect was reversed in the DSS + miR-4246 inhibitor + SIRT1-siRNA group compared with the DSS + miR-4246 inhibitor + control-siRNA group. The data revealed that miR-4246 functions as a marker in IBD progression. These results are consistent with those of previous studies. Sun et al (34) reported that miR-4262 regulated chondrocyte viability, apoptosis and autophagy by targeting SIRT1 and activating the PI3K/AKT/mTOR signaling pathway in rats with osteoarthritis. Accumulating studies have confirmed the role of $\mathrm{NF}-\kappa \mathrm{B}$ in inflammatory diseases $(35,36)$. Several reports have demonstrated that miRNAs activate the NF- $\kappa \mathrm{B}$ pathway, which may be associated with the development of inflammatory diseases $(37,38)$. Therefore, the present study further validated the effects of NF- $\kappa B$ activity in IBD. Notably, the present findings revealed that $\mathrm{p}-\mathrm{p} 65$ expression was increased and the ratio of p-p65/p65 was enhanced in DSS-treated Caco-2 cells. Transfection with the miR-4262 inhibitor knocked down p-p65 expression and decreased the ratio of p-p65/p65 in DSS-treated Caco-2 cells following transfection. However, opposite effects were observed in the DSS + miR-4246 inhibitor + SIRT1-siRNA group; the expression of p65 were enhanced and the ratio of p-p65/p65 was increased. These data demonstrated that miR-4246 inhibitor serves a protective role in IBD by alleviating apoptosis and activating NF- $\mathrm{KB}$ pathways.

Taken together, the present study, to the best of our knowledge, was the first to demonstrate the role of miR-4262 in the development of IBD. The results of the present study demonstrated the protective effects of transfection with miR-4262 inhibitor on DSS-induced Caco- 2 cells, evidenced by inhibiting the inflammatory response, increasing cell growth and decreasing cells apoptosis, via regulation of the miR-4262/SIRT1 axis. This indicates that miR-4262 may represent a potential target for IBD treatment.

\section{Acknowledgements}

Not applicable.

\section{Funding}

No funding was received.

\section{Availability of data and materials}

The datasets used and/or analyzed during the current study are available from the corresponding author on reasonable request.

\section{Authors' contributions}

XD contributed to study design, data collection, statistical analysis, data interpretation and manuscript preparation. LS, MD, LY and LX contributed to data collection and statistical analysis. XX contributed to data collection, statistical analysis, and manuscript preparation. All authors read and approved the final manuscript.

\section{Ethics approval and consent to participate}

All specimens were obtained with written informed consent and the present study was approved by the Ethics Committee of Chengdu Women's and Children's Central Hospital.

\section{Patient consent for publication}

Not applicable.

\section{Competing interests}

The authors declare that they have no competing interests.

\section{References}

1. Mogilevski T, Burgell R, Aziz Q and Gibson PR: Review article: The role of the autonomic nervous system in the pathogenesis and therapy of IBD. Aliment Pharmacol Ther 50: 720-737, 2019.

2. Hammer T, Lophaven SN, Nielsen KR, von Euler-Chelpin M, Weihe P, Munkholm P, Burisch J and Lynge E: Inflammatory bowel diseases in Faroese-born Danish residents and their offspring: Further evidence of the dominant role of environmental factors in IBD development. Aliment Pharmacol Ther 45: 1107-1114, 2017. 
3. Wolf DC, Abraham BP, Afzali A, Allegretti PD and Arai R Community perspectives: Combining serology, genetics, and inflammation markers for the diagnosis of IBD and differentiation between CD and UC. Gastroenterol Hepatol (N Y) 8: S1-S16, 2012.

4. Liu R, Tang A, Wang X, Chen X, Zhao L, Xiao Z and Shen S: Inhibition of lncRNA NEAT1 suppresses the inflammatory response in IBD by modulating the intestinal epithelial barrier and by exosome-mediated polarization of macrophages. Int J Mol Med 42: 2903-2913, 2018.

5. Pereira TDSF, Brito JAR, Guimarães ALS, Gomes CC de Lacerda JCT, de Castro WH, Coimbra RS, Diniz MG and Gomez RS: MicroRNA profiling reveals dysregulated microRNAs and their target gene regulatory networks in cemento-ossifying fibroma. J Oral Pathol Med 47: 78-85, 2018.

6. Wu ZW, Liu YF, Wang S and Li B: miRNA-146a induces vascular smooth muscle cell apoptosis in a rat model of coronary heart disease via NF-кB pathway. Genet Mol Res 14: 18703-18712, 2015.

7. Wang J, Yin K, Lv X, Yang Q, Shao M, Liu X and Sun H: MicroRNA-24-3p regulates Hodgkin's lymphoma cell proliferation, migration and invasion by targeting DEDD. Oncol Lett 17 365-371, 2019.

8. Chen Y, Du J, Zhang Z, Liu T, Shi Y, Ge X and Li YC: MicroRNA-346 mediates tumor necrosis factor $\alpha$-induced downregulation of gut epithelial vitamin $\mathrm{D}$ receptor in inflammatory bowel diseases. Inflamm Bowel Dis 20: 1910-1918, 2014.

9. Huang L, Ma Q, Li Y, Li B and Zhang L: Inhibition of microRNA-210 suppresses pro-inflammatory response and reduces acute brain injury of ischemic stroke in mice. Exp Neurol 300: 41-50, 2018.

10. Kumar A, Bhatia HS, de Oliveira AC and Fiebich BL: microRNA-26a modulates inflammatory response induced by toll-like receptor 4 stimulation in microglia. J Neurochem 135 1189-1202, 2015

11. Wang K, Ren Y, Liu Y, Zhang J and He JJ: miR-4262 promotes proliferation and invasion of human breast cancer cells through directly targeting KLF6 and KLF15. Oncol Res 25: 277-283, 2017.

12. Weng L, Ma J, Jia YP, Wu SQ, Liu BY, Cao Y, Yin X, Shang MY and Mao AW: miR-4262 promotes cell apoptosis and inhibits proliferation of colon cancer cells: Involvement of GALNT4. Am J Transl Res 10: 3969-3977, 2018.

13. Zhang D, Li Z, Zhang Y, Tu C, Huo J and Liu Y: miR-4262 promotes the proliferation of human cutaneous malignant melanoma cells through KLF6-mediated EGFR inactivation and p21 upregulation. Oncol Rep 36: 3657-3663, 2016.

14. Yang L, Duan Z, Liu X and Yuan Y: N-acetyl-l-cysteine ameliorates the PM2.5-induced oxidative stress by regulating SIRT-1 in rats. Environ Toxicol Pharmacol 57: 70-75, 2018.

15. Sim WC, Kim DG, Lee W, Sim H, Choi YJ and Lee BH: Activation of SIRT1 by L-serine increases fatty acid oxidation and reverses insulin resistance in $\mathrm{C} 2 \mathrm{C} 12$ myotubes. Cell Biol Toxicol 35: 457-470, 2019.

16. Pan Q, Lou X, Zhang J, Zhu Y, Li F, Shan Q, Chen X, Xie Y Su S, Wei H, et al: Genomic variants in mouse model induced by azoxymethane and dextran sodium sulfate improperly mimic human colorectal cancer. Sci Rep 7: 25, 2017.

17. Menconi A, Hernandez-Velasco X, Vicuña EA, Kuttappan VA, Faulkner OB, Tellez G, Hargis BM and Bielke LR: Histopathological and morphometric changes induced by a dextran sodium sulfate (DSS) model in broilers. Poult Sci 94: 906-911, 2015.

18. Nighot P, Young K, Nighot M, Rawat M, Sung EJ, Maharshak N, Plevy SE, Ma T and Blikslager A: Chloride channel $\mathrm{ClC}-2$ is a key factor in the development of DSS-induced murine colitis. Inflamm Bowel Dis 19: 2867-2877, 2013.

19. Livak KJ and Schmittgen TD: Analysis of relative gene expression data using real-time quantitative PCR and the 2(-Delta Delta C(T)) method. Methods 25: 402-408, 2001

20. Friedrich M, Pohin M and Powrie F: Cytokine networks in the pathophysiology of inflammatory bowel disease. Immunity 50 992-1006, 2019.
21. Rogler G: Resolution of inflammation in inflammatory bowel disease. Lancet Gastroenterol Hepatol 2: 521-530, 2017.

22. McDaniel DK, Eden K, Ringel VM and Allen IC: Emerging roles for noncanonical NF- $\kappa \mathrm{B}$ signaling in the modulation of inflammatory bowel disease pathobiology. Inflamm Bowel Dis 22: 2265-2279, 2016

23. Soufflet F, Biraud M, Rolli-Derkinderen M, Lardeux B, Trang C, Coron E, Bruley des Varannes S, Bourreille A and Neunlist M: Modulation of VIPergic phenotype of enteric neurons by colonic biopsy supernatants from patients with inflammatory bowel diseases: Involvement of IL-6 in Crohn's disease. Neurogastroenterol Motil 30, 2018.

24. Schenk M, Bouchon A, Seibold F and Mueller C: TREM-1-expressing intestinal macrophages crucially amplify chronic inflammation in experimental colitis and inflammatory bowel diseases. J Clin Invest 117: 3097-3106, 2007.

25. Zhao Q, Liu Y, Tan L, Yan L and Zuo X: Adiponectin administration alleviates DSS-induced colonic inflammation in Caco-2 cells and mice. Inflamm Res 67: 663-670, 2018.

26. Wirtz S, Neufert C, Weigmann B and Neurath MF: Chemically induced mouse models of intestinal inflammation. Nat Protoc 2 : 541-546, 2007.

27. Liu X, Chen J, Guan T, Yao H, Zhang W, Guan Z and Wang Y: Correction to: miRNAs and target genes in the blood as biomarkers for the early diagnosis of Parkinson's disease. BMC Syst Biol 13: 20, 2019.

28. Zhang J, Lian B, Shang Y, Li C and Meng Q: miR-135a protects dextran sodium sulfate-induced inflammation in human colorectal cell lines by activating STAT3 signal. Cell Physiol Biochem 51: 1001-1012, 2018.

29. Zhang H, Jiang H, Zhang H, Liu J, Hu X and Chen L: miR-4262, low level of which predicts poor prognosis, targets proto-oncogene CD163 to suppress cell proliferation and invasion in gastric cancer. Onco Targets Ther 12: 599-607, 2019.

30. Sun H, Zhou X, Bao Y, Xiong G, Cui Y and Zhou H: Involvement of miR-4262 in paclitaxel resistance through the regulation of PTEN in non-small cell lung cancer. Open Biol 9: 180227, 2019.

31. Song K, Liu N, Yang Y and Qiu X: Regulation of osteosarcoma cell invasion through osteopontin modification by miR-4262. Tumour Biol 37: 6493-6499, 2016.

32. Gerasimidis K, Edwards C, Stefanowicz F, Galloway P, McGrogan P, Duncan A and Talwar D: Micronutrient status in children with IBD: True deficiencies or epiphenomenon of the systemic inflammatory response. J Pediatr Gastroenterol Nutr 56: e50-e51, 2013.

33. Eichele DD and Kharbanda KK: Dextran sodium sulfate colitis murine model: An indispensable tool for advancing our understanding of inflammatory bowel diseases pathogenesis. World J Gastroenterol 23: 6016-6029, 2017.

34. Sun W, Li Y and Wei S: miR-4262 regulates chondrocyte viability, apoptosis, autophagy by targeting SIRT1 and activating $\mathrm{PI} 3 \mathrm{~K} / \mathrm{AKT} / \mathrm{mTOR}$ signaling pathway in rats with osteoarthritis. Exp Ther Med 15: 1119-1128, 2018.

35. Schuliga M: NF-kappaB signaling in chronic inflammatory airway disease. Biomolecules 5: 1266-1283, 2015.

36. Lin TH, Pajarinen J, Lu L, Nabeshima A, Cordova LA, Yao Z and Goodman SB: NF- $\kappa$ B as a therapeutic target in inflammatory-associated bone diseases. Adv Protein Chem Struct Biol 107: 117-154, 2017.

37. Hill JM, Clement $C$, Zhao $Y$ and Lukiw WJ: Induction of the pro-inflammatory NF-kB-sensitive miRNA-146a by human neurotrophic viruses. Front Microbiol 6: 43, 2015.

38. Pogue AI, Percy ME, Cui JG, Li YY, Bhattacharjee S, Hill JM, Kruck TP, Zhao Y and Lukiw WJ: Up-regulation of NF-kB-sensitive miRNA-125b and miRNA-146a in metal sulfate-stressed human astroglial (HAG) primary cell cultures. J Inorg Biochem 105: 1434-1437, 2011.

This work is licensed under a Creative Commons Attribution-NonCommercial-NoDerivatives 4.0 International (CC BY-NC-ND 4.0) License. 